\title{
Prognostic Value of Dual-Energy CT-Based Iodine Quantification versus Conventional CT in Acute Pulmonary Embolism: A Propensity-Match Analysis
}

Dong Jin Im, MD, Jin Hur, MD, PhD, Kyunghwa Han, PhD, Young Joo Suh, MD, PhD, Yoo Jin Hong, MD, PhD, Hye-Jeong Lee, MD, PhD, Young Jin Kim, MD, PhD, Byoung Wook Choi, MD, PhD All authors: Department of Radiology and Research Institute of Radiological Science, Severance Hospital, Yonsei University College of Medicine, Seoul, Korea

Objective: The present study aimed to investigate whether quantitative dual-energy computed tomography (DECT) parameters offer an incremental risk stratification benefit over the CT ventricular diameter ratio in patients with acute pulmonary embolism (PE) by using propensity score analysis.

Materials and Methods: This study was conducted on 480 patients with acute PE who underwent CT pulmonary angiography (CTPA) or DECT pulmonary angiography (DE CT-PA). This propensity-matched study population included 240 patients with acute PE each in the CTPA and DECT groups. Altogether, 260 (54.1\%) patients were men, and the mean age was 64.9 years (64.9 \pm 13.5 years). The primary endpoint was all-cause death within 30 days. The Cox proportional hazards regression model was used to identify associations between $\mathrm{CT}$ parameters and outcomes and to identify potential predictors. Concordance (C) statistics were used to compare the prognoses between the two groups.

Results: In both CTPA and DECT groups, right to left ventricle diameter ratio $\geq 1$ was associated with an increased risk of allcause death within 30 days (hazard ratio: 3.707, $p<0.001$ and 5.573, $p<0.001$, respectively). However, C-statistics showed no statistically significant difference between the CTPA and DECT groups for predicting death within 30 days (C-statistics: 0.759 vs. $0.819, p=0.117$ ).

Conclusion: Quantitative measurement of lung perfusion defect volume by DECT had no added benefit over CT ventricular diameter ratio for predicting all-cause death within 30 days.

Keywords: Acute pulmonary embolism; Dual-energy computed tomography (DECT); Ventricular diameter; Lung perfusion

\section{INTRODUCTION}

Risk stratification is important in patients with acute pulmonary embolism (PE) because optimal management, monitoring, and therapeutic strategies depend on the prognosis $(1,2)$. Many computed tomography (CT)

Received: August 28, 2019 Revised: February 26, 2020

Accepted: March 9, 2020

Corresponding author: Jin Hur, MD, PhD, Department of Radiology and Research Institute of Radiological Science, Severance Hospital, Yonsei University College of Medicine, 50-1 Yonsei-ro, Seodaemungu, Seoul 03722, Korea.

- E-mail: khuhz@yuhs.ac

This is an Open Access article distributed under the terms of the Creative Commons Attribution Non-Commercial License (https://creativecommons.org/licenses/by-nc/4.0) which permits unrestricted non-commercial use, distribution, and reproduction in any medium, provided the original work is properly cited. parameters have been proposed as potential predictors of $\mathrm{PE}$ severity and clinical outcome. Currently, the CT ventricular diameter (VD) ratio is a well-established and widely used prognostic indicator in patients with acute PE. A previous meta-analysis demonstrated that the quantitative CT parameter of right to left ventricle diameter ratio greater than 1 showed the strongest predictive ability and most robust evidence for an adverse clinical outcome in patients with acute PE (3).

Dual-energy computed tomography (DECT) has been used in the diagnosis and evaluation of $\mathrm{PE}$, and recent studies have shown that quantitative parameters of DECT are helpful in predicting the clinical outcome of patients with PE (4-13). A previous study demonstrated that DECT perfusion imaging could display pulmonary perfusion defects with good agreement to scintigraphic findings (5). 
Several studies have described the functional relevance of perfusion defects (PDs) detected on DECT, and studies have shown that the extent of PDs measured with DECT correlates with an adverse clinical outcome in patients with PE (9, 10). Studies demonstrating the clinical utility of PDs using DECT have been published, but there is little evidence for the additional risk stratification benefit of the CT VD ratio in patients with acute PE (8-11).

The purpose of the present study was to investigate whether quantitative DECT parameters offer incremental risk stratification benefits over the CT VD ratio in patients with acute PE by using a propensity score analysis.

\section{MATERIALS AND METHODS}

\section{Patient Population}

This single-center, propensity score-matched study compared the predictive value of quantitative DECT parameters and CT VD ratio in patients with acute PE. Institutional Review Board approval was obtained, and the requirement for informed consent was waived for this retrospective propensity score-matched study.

All consecutive patients who underwent CT pulmonary angiography (CTPA) or DECT pulmonary angiography (DE CT$\mathrm{PA}$ ) and were suspected to have acute PE between January 2015 and December 2017 were considered potentially eligible for this analysis. Among 3419 patients (CTPA group, $n=2045$, DECT group, $n=1374$ ), the following patients were excluded: patients with negative CT results $(n=$ 2486), patients who did not clinically or radiologically meet the criteria for acute PE $(n=47)$, those in whom DECT or CT was performed as a follow-up CT examination after receiving anticoagulation therapy $(n=105)$, and those for whom CT image quality was insufficient or CT image data were not available $(n=21)$. Finally, 484 patients $(23.6 \%)$ who were diagnosed with acute PE by CTPA and 276 patients (20.1\%) who were diagnosed with acute PE by DECT were recruited for the present study (Fig. 1).

Patient clinical information, including age, sex, and medical history (hypertension, diabetes mellitus, smoking, heart disease [including congenital heart disease, coronary artery disease, myocardial infarction, valvular heart disease, heart failure, arrhythmia and cardiomyopathy], chronic obstructive pulmonary disease [COPD], pneumonia, history of cancer, history of deep vein thrombosis [DVT]), was recorded based on patient medical records.

To reduce potential selection bias related to the use

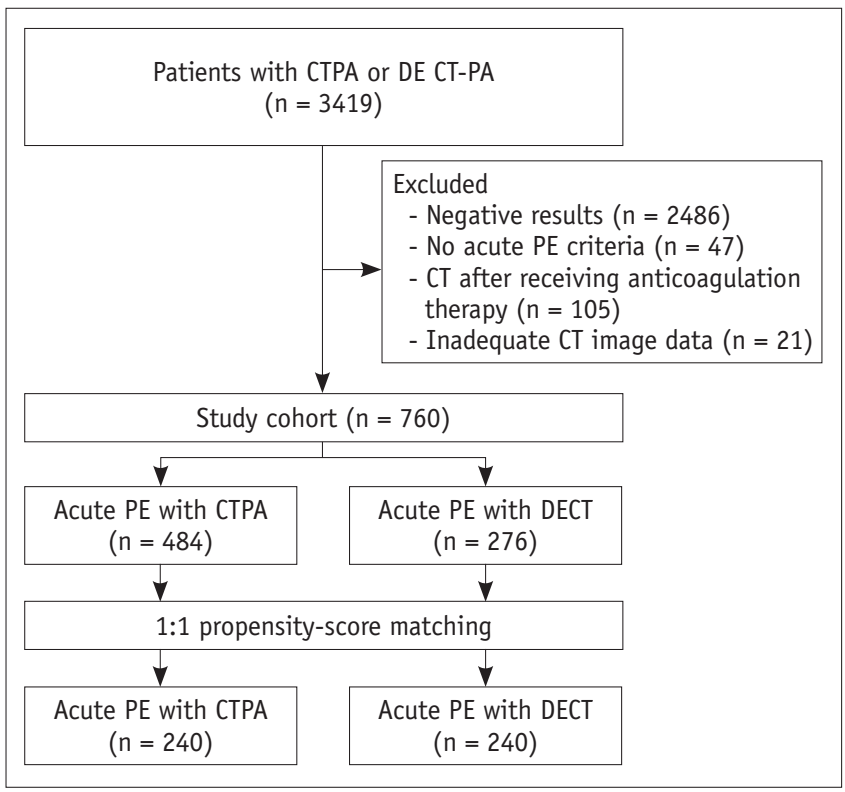

Fig. 1. Flowchart of patient selection. $C T P A=C T$ pulmonary angiography, $\mathrm{DECT}=$ dual-energy $\mathrm{CT}, \mathrm{DE} \mathrm{CT}-\mathrm{PA}=\mathrm{DECT}$ pulmonary angiography, $\mathrm{PE}=$ pulmonary embolism

of a non-randomized cohort to generate two groups (CTPA and DECT groups) with comparable characteristics, propensity score-matched analyses were performed (14). The following variables were used to develop the propensity score and create a well-matched control group: age, sex, hypertension, diabetes mellitus, smoking, heart disease, COPD, pneumonia, history of cancer, and history of DVT. The balance of covariates between the groups was assessed by the absolute standardized mean difference before and after the matching procedure. An absolute standardized mean difference of 0.1 or less indicates balanced covariates between the two groups (15).

The propensity-matched study population included 240 patients with acute PE in the CTPA group and 240 patients with acute PE in the DECT group. Altogether, 260 (54.1\%) were men, and the mean age was 64.9 years $(64.9 \pm 13.5)$.

\section{CT Examination}

CTPA was performed for all participants by using a 64or 128-channel CT system (Revolution EVO, GE Healthcare, Chicago, IL, USA or Somatom Definition AS, Siemens Healthineers, Forchheim, Germany), and DE CT-PA was performed for all participants by using a dual-source CT system (Somatom Definition Flash, Siemens Healthineers). All patients received $50-90 \mathrm{~mL}$ of iopamidol $(370 \mathrm{mg} /$ $\mathrm{mL}$ iodine, Pamiray 370, Dongkook Pharmaceutical, Seoul, Korea) via an antecubital vein at $4 \mathrm{~mL} / \mathrm{s}$ by a power 
injector. Following contrast injection, $30 \mathrm{~mL}$ of saline was administered. During the scan, patients held their breath on inspiration. Pulmonary trunk attenuation was tracked by a bolus-tracking technique. Image acquisition was triggered manually once attenuation in the pulmonary trunk reached 100 Hounsfield units (HU). Radiation exposure was estimated from the dose-length product (DLP). The calculated mean radiation dose was $5.2 \mathrm{mSv}$ (DLP range, $189-903 \mathrm{mGy}^{*} \mathrm{~cm}$ ) based on the scan range and patient body weight.

\section{Image Analysis}

A radiologist with over 10 years of experience in chest CT

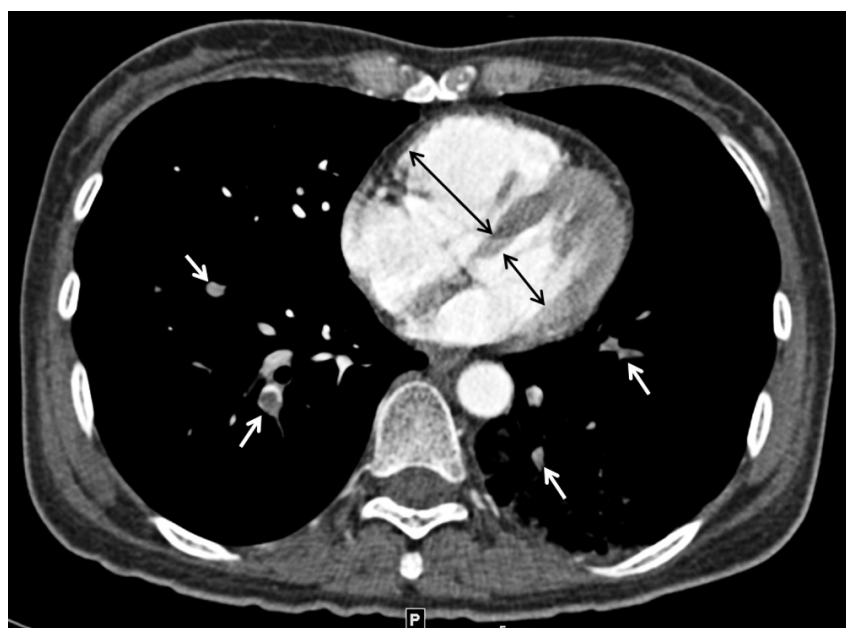

Fig. 2. Measurement of ventricular diameter ratio in 45-yearold woman with acute pulmonary embolism. Axial CT image shows measurement of maximum diameters (black arrows) of right and left ventricles. Ventricular diameter is maximal distance between ventricular endocardium and interventricular septum perpendicular to long axis of heart. White arrows indicate pulmonary embolism. analysis analyzed the CT data; the radiologist was blinded to patient identities and clinical histories. All scans were processed and read using a dedicated workstation equipped with dual-energy post-processing software (Syngo MMWP VE36A, Siemens Healthineers). The weighted average image was approximately $120 \mathrm{kV}$ and was automatically generated from a combination of the $140-\mathrm{kV}$ and $100-\mathrm{kV}$ data used for DE CT-PA. Color-coded iodine maps were merged with the corresponding $\mathrm{CT}$ angiographic images with soft tissue settings to create fusion images, allowing simultaneous depiction of occluded PAs and lung perfusion.

For quantitative analysis, maximal diameters of the right and left ventricles (RV and LV) were measured on transverse sections by identifying the maximal distance between the ventricular endocardium and the interventricular septum perpendicular to the long axis of the heart (Fig. 2). RV/LV diameter ratios were calculated by dividing the maximum diameters of the RV and LV. PD volume was analyzed and quantified from iodine maps by using dedicated Volume analysis software (version VE36A, Siemens Healthineers). $\mathrm{PD}$ attenuation values were measured automatically from -1 to $-1024 \mathrm{HU}$ in $\mathrm{HU}$ (Fig. 3). Total lung volume was analyzed by Lung Parenchyma Analysis (Syngo InSpace, Siemens Healthineers) and measured automatically by determining the sum of values from 1024 to $1 \mathrm{HU}$ and from -1 to $-1024 \mathrm{HU}$. The trachea and bronchus were excluded by a semiautomatic segmentation technique. The PDs values measured on iodine maps were carefully reviewed and compared to CT findings. PDs related to lung parenchymal abnormalities (e.g., infiltration, effusion, or emphysema) were manually excluded. The relative perfusion defect
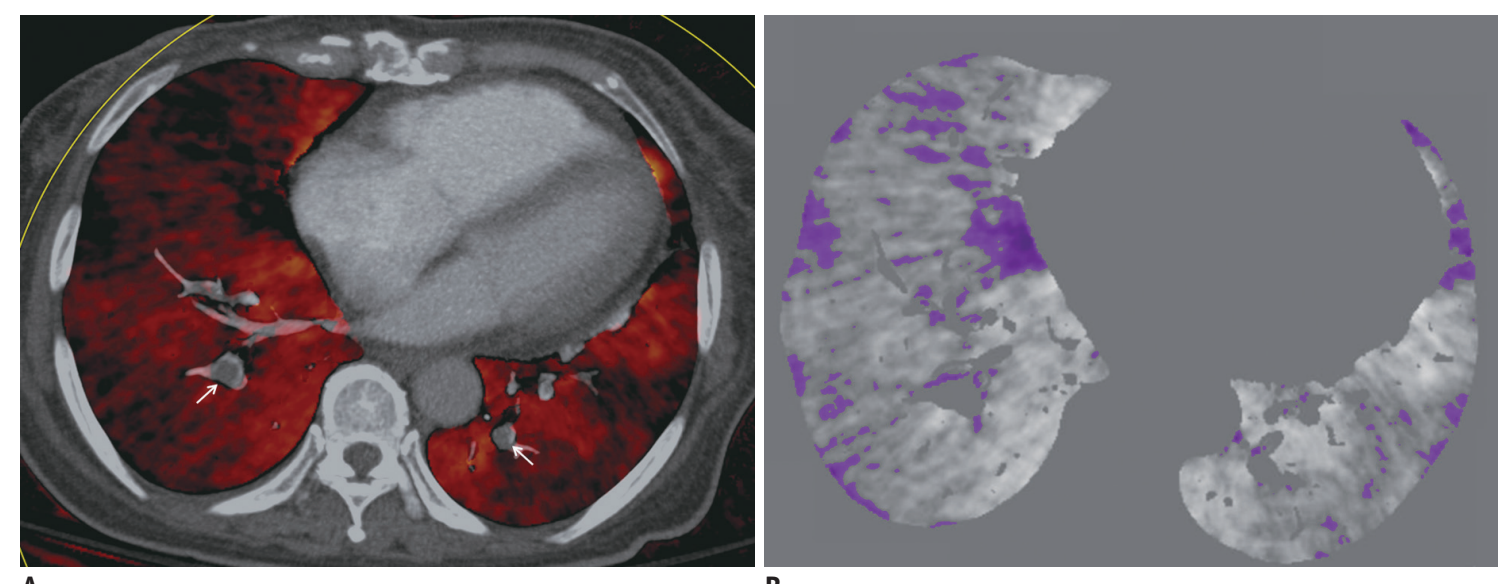

A

B

Fig. 3. 68-year-old woman with acute pulmonary embolism.

A. Iodine map generated on axial CT image with dedicated software shows pulmonary embolism (arrows) and perfusion defects in both lungs.

B. Map obtained with volume analysis software shows perfusion defect volume, measured from -1024 to $-1 \mathrm{HU}$, of $261.46 \mathrm{~cm}^{3}$ and relative perfusion defect volume of $11.4 \%$. 
volume (RelPD\%) was calculated as follows: RelPD\% $=$ PD volume / total lung volume $\times 100$. To assess the interobserver agreement for quantitative measurements, 50 of 240 patients in the DECT group were randomly selected and an independent reviewer with over 5 years of experience in chest $\mathrm{CT}$ analysis measured the PD volume and ventricular ratios.

\section{Clinical Outcome}

Clinical outcome data were obtained via a review of the electronic medical records or by telephone contact from a dedicated research nurse who was blinded to the CT results. The primary endpoints of the present study were death within 30 days from any cause. Patient death status was ascertained by querying the National Health Insurance Corporation.

\section{Statistical Analysis}

An analytic sample was created using propensity scorebased matching to correct for differences in patient characteristics in the two groups. Propensity score matching was conducted in a 1:1 ratio by nearest neighbor matching. The adequacy of the propensity model was confirmed by checking the covariate balance before and after matching.

Comparisons between the CTPA and DECT groups were performed. The differences between categorical variables were analyzed by chi-squared test or Fisher's exact test. The differences between continuous variables were analyzed by the Shapiro-Wilk test or Mann-Whitney U test. A Cox proportional hazards regression model was used to identify associations between $\mathrm{CT}$ parameters and outcomes and to identify potential predictors. Only variables with $p$ values less than 0.20 in univariate analyses were added to the final multivariate models to prevent model over-fitting. From the Cox proportional hazards model, hazard ratios (HRs) and 95\% confidence intervals (CIs) were calculated. Concordance (C) statistics were used to compare the predictive prognosis between the two groups. Inter-observer agreement was tested using intraclass correlation coefficients (ICCs). A $p$ value $<0.05$ was considered statistically significant. All statistical analyses were performed using $\mathrm{R}$ (version 3.2.2., R Foundation for Statistical Computing, Vienna, Austria).

\section{RESULTS}

\section{Baseline Clinical and CT Characteristics}

Baseline characteristics of the two groups are shown in Table 1. Both groups were matched for baseline variables, and no significant differences were observed for any of the baseline comparisons.

In the CTPA group, patients who died showed a higher prevalence of pneumonia and cancer (all $p<0.05$ ). In the DECT group, patients who died showed a higher prevalence of pneumonia, cancer, and DVT (all $p<0.05$ ). Other clinical characteristics were not significantly different between patients who survived and those who died (Table 2).

In both groups, VD ratios (1.10 vs. $0.97 ; p<0.001$ and 1.09 vs. $0.94 ; p<0.001)$ were significantly higher in

Table 1. Patient Clinical Characteristics before and after Propensity Score Matching

\begin{tabular}{|c|c|c|c|c|c|c|}
\hline \multirow[b]{2}{*}{ Characteristics } & \multicolumn{3}{|c|}{ Before Matching } & \multicolumn{3}{|c|}{ After Matching } \\
\hline & $\begin{array}{l}\text { CTPA Group } \\
(\mathrm{n}=484)\end{array}$ & $\begin{array}{l}\text { DECT Group } \\
(n=276)\end{array}$ & $\begin{array}{c}\text { Standardized } \\
\text { Difference }\end{array}$ & $\begin{array}{l}\text { CTPA Group } \\
(n=240)\end{array}$ & $\begin{array}{c}\text { DECT Group } \\
(n=240)\end{array}$ & $\begin{array}{c}\text { Standardized } \\
\text { Difference }\end{array}$ \\
\hline Age (years) & $67.8 \pm 15.4$ & $64.6 \pm 13.9$ & -0.227 & $64.3 \pm 15.8$ & $65.4 \pm 13.5$ & 0.079 \\
\hline Sex (female) & $268(55.4)$ & $150(54.3)$ & -0.021 & $128(52.9)$ & $132(54.5)$ & 0.033 \\
\hline \multicolumn{7}{|l|}{ Clinical condition } \\
\hline Hypertension & $213(44.0)$ & $137(49.6)$ & 0.112 & $118(48.8)$ & $118(48.8)$ & 0 \\
\hline Diabetes mellitus & $88(18.2)$ & $66(23.9)$ & 0.134 & 53 (21.9) & $54(22.3)$ & 0.009 \\
\hline Smoking* & $117(24.2)$ & $57(20.7)$ & -0.086 & $55(22.7)$ & $53(21.9)$ & -0.020 \\
\hline Heart disease $^{\dagger}$ & $26(5.4)$ & $29(10.5)$ & 0.167 & $16(6.6)$ & $21(8.7)$ & 0.067 \\
\hline COPD & $30(6.2)$ & $20(7.2)$ & 0.040 & $19(7.9)$ & $19(7.9)$ & 0 \\
\hline Pneumonia & $63(13.0)$ & $38(13.8)$ & 0.021 & $33(13.6)$ & $33(13.6)$ & 0 \\
\hline Cancer & $206(42.6)$ & $160(58.0)$ & 0.311 & $126(52.1)$ & $131(54.1)$ & 0.041 \\
\hline DVT & $57(11.8)$ & 77 (27.9) & 0.358 & $48(19.8)$ & $48(19.8)$ & 0 \\
\hline
\end{tabular}

Values are presented as mean \pm standard deviation or patient number with (\%). ${ }^{*}$ Current or former smoker, ${ }^{\dagger}$ Heart disease includes congenital heart disease, coronary artery disease, myocardial infarction, valvular heart disease, heart failure, arrhythmia and cardiomyopathy. $\mathrm{COPD}=$ chronic obstructive pulmonary disease, $\mathrm{CTPA}=\mathrm{CT}$ pulmonary angiography, $\mathrm{DECT}=$ dual-energy $\mathrm{CT}$, DVT $=$ deep vein thrombosis 
the death group than in the survival group. In the DECT group, the RelPD\% (10.21\% vs. $7.73 \% ; p<0.001)$ was also significantly higher in the death group than in the survival group (Table 2).

\section{Clinical and СТ Variables Associated with Outcome}

During the median follow-up period of 133 days (interquartile range: $35-401$ days), there were 35 deaths within 30 days from any cause in the CTPA group and 45 deaths within 30 days from any cause in the DECT group.

In univariate analysis using a Cox hazards regression model in the CTPA group, pneumonia and cancer were predictors of all-cause death within 30 days (all, $p<0.05$ ) (Table 3). Patients with a larger VD ratio $(\geq 1$ vs. $<1)$ had a significantly higher risk of death within 30 days $(p=0.001)$ (Table 3). In the DECT group, univariate analysis using a Cox hazards regression model revealed that pneumonia, cancer, and DVT were predictors of all-cause death within 30 days (all, $p<0.05)$. Patients with a larger VD ratio $(\geq 1$ vs. $<1$ ) and a larger PD volume had a significantly higher risk of death within 30 days (all $p<0.001$ ) (Table 3 ).

In multivariate analysis using a Cox hazards regression model adjusted for age, pneumonia, cancer, and VD ratio $(\geq 1)$ were associated with an increased risk of death within 30 days. In the CTPA group, pneumonia, cancer, and VD ratio $(\geq 1)$ (HR, 3.707; 95\% CI, 1.730-7.941; $p$ $<0.001$ ) were associated with an increased risk of death within 30 days. In the DECT group, pneumonia, cancer, VD ratio $(\geq 1)(H R, 5.573 ; 95 \% C I, 2.758-11.261 ; p<0.001)$ and RelPD\% (HR, 1.038; 95\% CI, 1.005-1.072; $p=0.022$ ) were associated with an increased risk of death within 30 days. The C-statistics showed no statistically significant difference between the CTPA and DECT groups for predicting death within 30 days (C-statistics: 0.759 vs. $0.819, p=$ 0.117) (Table 4).

There was excellent inter-observer agreement between the two radiologists in the measurement parameters of DECT. The ICCs for RelPD\% and VD ratios were 0.88 (95\% CI: $0.81-0.97)$ and 0.93 (95\% CI: $0.89-0.99)$, respectively.

\section{DISCUSSION}

This study was designed to investigate whether quantitative DECT parameters provide incremental risk

Table 2. Baseline Characteristics of Matched Study Population according to Mortality Status

\begin{tabular}{|c|c|c|c|c|c|c|}
\hline \multirow{2}{*}{ Characteristics } & \multicolumn{3}{|c|}{ CTPA Group } & \multicolumn{3}{|c|}{ DECT Group } \\
\hline & Survivor $(n=207)$ & Death $(n=35)$ & $P$ & Survivor $(n=197)$ & Death $(n=45)$ & $P$ \\
\hline Age (years) & $65(58,78)$ & $67(55,75)$ & 0.115 & $69(57,74)$ & $70(62,74)$ & 0.148 \\
\hline Sex (female) & $107(51.7)$ & $21(60.0)$ & 0.362 & $111(56.3)$ & $21(46.7)$ & 0.239 \\
\hline \multicolumn{7}{|l|}{ Clinical condition } \\
\hline Hypertension & $98(47.3)$ & $20(57.1)$ & 0.283 & $91(46.2)$ & $27(60.0)$ & 0.094 \\
\hline Diabetes mellitus & $44(21.3)$ & $9(25.7)$ & 0.555 & $42(21.3)$ & $12(26.7)$ & 0.437 \\
\hline Smoking* & $47(22.7)$ & $8(22.9)$ & 0.984 & $42(21.3)$ & $11(24.4)$ & 0.647 \\
\hline Heart disease $^{\dagger}$ & $13(6.3)$ & $3(8.6)$ & 0.613 & $17(8.6)$ & $4(8.9)$ & 0.956 \\
\hline COPD & $14(6.8)$ & $5(14.3)$ & 0.126 & $13(6.6)$ & $6(13.3)$ & 0.129 \\
\hline Pneumonia & $24(11.6)$ & $9(25.7)$ & 0.024 & $18(9.1)$ & $15(33.3)$ & $<0.001$ \\
\hline Cancer & $99(47.8)$ & $27(77.1)$ & 0.001 & $96(48.7)$ & $35(77.8)$ & 0.001 \\
\hline DVT & $44(21.3)$ & $4(11.4)$ & 0.175 & $45(22.8)$ & $3(6.7)$ & 0.014 \\
\hline \multicolumn{7}{|l|}{ Treatment } \\
\hline Anticoagulants & $177(85.5)$ & $26(74.2)$ & 0.150 & $159(80.7)$ & $33(73.3)$ & 0.367 \\
\hline Thrombolytic treatment & $6(2.8)$ & $2(5.7)$ & 0.703 & $5(2.5)$ & $2(4.4)$ & 0.845 \\
\hline Inferior vena cava filter & $22(10.6)$ & 0 & 0.088 & $23(11.6)$ & $1(2.2)$ & 0.102 \\
\hline \multicolumn{7}{|l|}{ CT measurement } \\
\hline Ventricular diameter ratio, median & $0.97(0.87,1.26)$ & $1.10(0.98,1.24)$ & $<0.001$ & $0.94(0.87,1.01)$ & $1.09(0.99,1.32)$ & $<0.001$ \\
\hline Ventricular diameter ratio $(\geq 1)$ & $89(43.0)$ & $26(74.3)$ & $<0.001$ & $53(26.9)$ & $33(73.3)$ & $<0.001$ \\
\hline $\begin{array}{l}\text { Relative perfusion defect } \\
\text { volume }(\%) \text {, median }\end{array}$ & - & - & - & $\begin{array}{c}7.73 \\
(5.04,12.49)\end{array}$ & $\begin{array}{c}10.21 \\
(6.88,13.87)\end{array}$ & $<0.001$ \\
\hline
\end{tabular}

Values are presented as median value (1st quantile, 3rd quantile) or patient number (\%). ${ }^{*}$ Current or former smoker, ${ }^{\dagger}$ Heart disease includes congenital heart disease, coronary artery disease, myocardial infarction, valvular heart disease, heart failure, arrhythmia and cardiomyopathy. 
Table 3. Univariate Analysis Using Cox Proportional Hazards Regression Model

\begin{tabular}{|c|c|c|c|c|c|c|}
\hline \multirow{2}{*}{ Characteristics } & \multicolumn{3}{|c|}{ CTPA Group } & \multicolumn{3}{|c|}{ DECT Group } \\
\hline & HR & $95 \% \mathrm{CI}$ & $P$ & HR & $95 \% \mathrm{CI}$ & $P$ \\
\hline Age & 1.009 & $0.987-1.032$ & 0.417 & 1.024 & $0.998-1.049$ & 0.065 \\
\hline Sex (female) & 1.317 & $0.670-2.590$ & 0.424 & 0.672 & $0.374-1.207$ & 0.283 \\
\hline Hypertension & 1.442 & $0.738-2.817$ & 0.283 & 1.616 & $0.890-2.934$ & 0.115 \\
\hline Diabetes mellitus & 1.277 & $0.598-2.726$ & 0.526 & 1.289 & $0.666-2.496$ & 0.451 \\
\hline Smoking* & 0.992 & $0.451-2.183$ & 0.983 & 1.180 & $0.598-2.330$ & 0.632 \\
\hline Heart disease $^{\dagger}$ & 1.387 & $0.425-4.531$ & 0.587 & 1.256 & $0.450-3.510$ & 0.663 \\
\hline COPD & 2.082 & $0.807-5.366$ & 0.129 & 2.177 & $0.921-5.144$ & 0.076 \\
\hline Pneumonia & 2.554 & $1.196-5.451$ & 0.015 & 3.949 & $2.120-7.357$ & $<0.001$ \\
\hline Cancer & 3.329 & $1.512-7.329$ & 0.002 & 3.413 & $1.689-6.895$ & $<0.001$ \\
\hline DVT & 0.502 & $0.177-1.423$ & 0.195 & 0.260 & $0.081-0.840$ & 0.024 \\
\hline Anticoagulants & 1.787 & $0.774-4.072$ & 0.278 & 2.104 & $0.813-5.062$ & 0.181 \\
\hline Thrombolytic treatment & 0.518 & $0.321-0.947$ & 0.186 & 0.584 & $0.238-1.142$ & 0.238 \\
\hline Inferior vena cava filter & 2.031 & $0.781-9.712$ & 0.142 & 2.579 & $0.744-10.164$ & 0.115 \\
\hline VD ratio $(\geq 1)$ & 3.407 & $1.596-7.270$ & 0.001 & 7.471 & $3.593-15.534$ & $<0.001$ \\
\hline RelPD\% & - & - & - & 1.065 & $1.013-1.120$ & 0.012 \\
\hline
\end{tabular}

${ }^{*}$ Current or former smoker, ${ }^{\dagger}$ Heart disease includes congenital heart disease, coronary artery disease, myocardial infarction, valvular heart disease, heart failure, arrhythmia and cardiomyopathy. Dash (-) indicates no patient. CI = confidence interval, HR $=$ hazard ratio, RelPD\% $=$ relative perfusion defect volume, $V D=$ ventricular diameter

Table 4. Multivariate Analysis Using Cox Proportional Hazards Regression Model

\begin{tabular}{|c|c|c|c|c|c|c|}
\hline \multirow{2}{*}{ Characteristics } & \multicolumn{3}{|c|}{ CTPA Group } & \multicolumn{3}{|c|}{ DECT Group } \\
\hline & HR & $95 \% \mathrm{CI}$ & $P$ & HR & $95 \% \mathrm{CI}$ & $P$ \\
\hline Age & 1.002 & $0.961-1.024$ & 0.817 & 1.032 & $1.004-1.065$ & 0.260 \\
\hline COPD & 2.064 & $0.793-5.370$ & 0.137 & 1.578 & $0.624-3.908$ & 0.342 \\
\hline Pneumonia & 2.092 & $0.971-4.509$ & 0.049 & 2.178 & $1.102-4.288$ & 0.025 \\
\hline Cancer & 3.111 & $1.380-7.013$ & 0.006 & 3.972 & $1.936-8.147$ & $<0.001$ \\
\hline DVT & 0.739 & $0.255-2.141$ & 0.577 & 0.513 & $0.153-1.718$ & 0.282 \\
\hline VD ratio $(\geq 1$ vs. $<1)$ & 3.707 & $1.730-7.941$ & $<0.001$ & 5.573 & $2.758-11.261$ & $<0.001$ \\
\hline RelPD\% & - & - & - & 1.038 & $1.005-1.072$ & 0.022 \\
\hline Concordance-index & & 0.759 & & & 0.819 & \\
\hline
\end{tabular}

Dash (-) indicates no patient.

stratification benefits over the CT VD ratio in patients with acute PE by using a propensity score analysis. Based on this study, quantitative measurement of lung PD volume by DECT offered no added benefit over CT VD ratio for predicting allcause death within 30 days.

Risk stratification for patients with acute PE is important to establish appropriate treatment and management. CT parameters have emerged as prognostic markers to assess the severity of hemodynamic compromise from acute PE and identify patients at heightened risk for fatal or nonfatal adverse events, thus guiding clinical management $(3,16,17)$. For clinical purposes, the RV/LV diameter ratio measured on $\mathrm{CT}$ shows the strongest predictive value across all endpoints and provides the most robust evidence for adverse clinical outcomes in patients with acute PE.
Many studies have supported that RV dysfunction assessed on CT was associated with an increased risk of early complications, including all-cause death and PE-related serious adverse events (16-22). In addition, previous metaanalyses have demonstrated that increased RV/LV diameter ratio is the strongest predictor of adverse clinical outcomes in patients with acute $\operatorname{PE}(3,21,22)$. This measurement is a simple quantitative value that can be easily measured in axial or 4-chamber images using СT. Our results are in agreement with those of previous studies. In our study, right ventricular dysfunction was assessed by $\mathrm{CT}$ by using two-dimensional axial transverse images. According to our study, right ventricular dysfunction on CT was an independent predictor of all-cause death within 30 days.

DECT has been proposed as a new imaging technique 
for detecting PE $(11,12)$. A unique feature of DECT is that it allows differentiation of materials based on their energy absorption $(4,23)$. Thus, DECT allows simultaneous assessment of pulmonary vasculature and parenchymal iodine distribution $(5,6,24)$. In the lung, the pattern of iodine enhancement on DECT has been shown to correspond to lung blood volume on planar scintigraphy (5). Several studies have reported that the quantitative values of lung PDs on DECT correlated with right ventricular dysfunction and adverse clinical outcomes (8-11). A previous study reported that the extent of lung PDs on DECT correlated well with right ventricular dysfunction on $\mathrm{CT}$ and death (9). Another study demonstrated that of all evaluated CT parameters, the PD volume measured by DECT showed the highest predictive power for detecting an adverse clinical outcome (10). Conversely, our previous study revealed that lung PDs quantified on DECT had no added benefit in predicting death within 30 days or for predicting PE-related death (11). Based on previous studies, quantitative DECT parameters have potential for use as prognostic makers in acute PE. However, the value of quantitative DECT parameters for prognosis and risk stratification in acute PE is controversial. The heterogeneity of study groups, definitions, and outcomes prohibits consensus on the prognostic performance of DECT.

We conducted a propensity score-matched study to compare the predictive value of quantitative DECT parameters and CT VD ratio in patients with acute PE. Propensity score adjustment is a method of balancing the distribution of biases and confounders between groups, thereby increasing between-group comparability. Propensity score analysis is increasingly being applied as a statistical method in observational studies (14). We constructed two models to evaluate the added value of DECT parameters (lung PD volume) in predicting all-cause death within 30 days. Although PDs measured on DECT were associated with an increased risk of death within 30 days, C-statistics showed no statistically significant difference between the two groups (CTPA group and DECT group) in predictive prognosis with respect to predicting death in patients with acute PE. These results suggest that DECT parameters (lung PD) had no added benefit over the simple quantified CT value of VD ratio for predicting death within 30 days in patients with acute PE.

Lung perfusion imaging is based on quantification of tissue enhancement at serial time points following contrast administration. Previous studies have demonstrated that the extent of PDs, identified by perfusion scintigraphy, correlated with clinical outcomes in patients with PE (25, 26). Consequently, the extent of PDs quantified on DECT is potentially predictive of hemodynamic changes in acute $\mathrm{PE}$. Thus, these are emerging as imaging biomarkers for risk stratification. However, there are several issues regarding quantitative measurements in DECT. First, DECT scans are usually obtained at a single time-point, so DECT provides an iodine distribution map of the lung microcirculation at a given time point (27). Therefore, quantitative DECT parameters can vary according to different clinical settings and with different imaging protocols. Second, there is no standardized analytical method for lung PDs using DECT in terms of HU threshold and analytical software. In addition, additional time is required to analyze lung perfusion using special software.

Our study has certain limitations. First, this study was conducted at a single center with a modest sample size. In addition, the retrospective nature of this study may be associated with a selection bias. However, we conducted a propensity score-matched study to balance the distribution of biases and confounders between groups. Second, the imaging protocol and analytical method for lung perfusion may have significantly influenced the results. Currently, there is no standardized analytical method for assessing lung PDs using DECT in terms of HU threshold and analytical software.

In conclusion, an increased RV/LV diameter ratio was associated with increased risk of all-cause death within 30 days in patients with acute PE. However, quantitative measurement of lung PD volume by DECT offered no added benefit over CT VD ratio in predicting all-cause death within 30 days. Our present data failed to provide an additional benefit of functional lung assessment on DECT for predicting future death in patients with PE. Future large trials with much longer follow-up periods must be performed to estimate the potential influence of DECT findings on treatment strategies and optimize the management and outcome of patients with acute PE.

\section{Conflicts of Interest}

The authors have no potential conflicts of interest to disclose.

\section{ORCID iDs}

Jin Hur

https://orcid.org/0000-0002-8651-6571 
Dong Jin Im

https://orcid.org/0000-0001-8139-5646

\section{REFERENCES}

1. Konstantinides S, Goldhaber SZ. Pulmonary embolism: risk assessment and management. Eur Heart J 2012;33:3014-3022

2. Remy-Jardin M, Pistolesi M, Goodman LR, Gefter WB, Gottschalk A, Mayo JR, et al. Management of suspected acute pulmonary embolism in the era of CT angiography: a statement from the Fleischner Society. Radiology 2007;245:315-329

3. Meinel FG, Nance JW Jr, Schoepf UJ, Hoffmann VS, Thierfelder $K M$, Costello $P$, et al. Predictive value of computed tomography in acute pulmonary embolism: systematic review and meta-analysis. Am J Med 2015;128:747-759.e2

4. Johnson TR, Krauss B, Sedlmair M, Grasruck M, Bruder H, Morhard D, et al. Material differentiation by dual energy CT: initial experience. Eur Radiol 2007;17:1510-1517

5. Thieme SF, Becker CR, Hacker M, Nikolaou K, Reiser MF, Johnson TR. Dual energy CT for the assessment of lung perfusion-Correlation to scintigraphy. Eur J Radiol 2008;68:369-374

6. Thieme SF, Johnson TR, Lee C, McWilliams J, Becker CR, Reiser $M F$, et al. Dual-energy CT for the assessment of contrast material distribution in the pulmonary parenchyma. AJR Am J Roentgenol 2009;193:144-149

7. Ferda J, Ferdová E, Mírka H, Baxa J, Bednářová A, Flohr T, et al. Pulmonary imaging using dual-energy $\mathrm{CT}$, a role of the assessment of iodine and air distribution. Eur $\mathrm{J}$ Radiol 2011;77:287-293

8. Chae EJ, Seo JB, Jang YM, Krauss B, Lee CW, Lee HJ, et al. Dual-energy CT for assessment of the severity of acute pulmonary embolism: pulmonary perfusion defect score compared with CT angiographic obstruction score and right ventricular/left ventricular diameter ratio. AJR Am J Roentgenol 2010;194:604-610

9. Bauer RW, Frellesen C, Renker M, Schell B, Lehnert T, Ackermann $\mathrm{H}$, et al. Dual energy CT pulmonary blood volume assessment in acute pulmonary embolism-Correlation with D-dimer level, right heart strain and clinical outcome. Eur Radiol 2011;21:1914-1921

10. Apfaltrer P, Bachmann V, Meyer M, Henzler T, Barraza JM, Gruettner J, et al. Prognostic value of perfusion defect volume at dual energy CTA in patients with pulmonary embolism: correlation with CTA obstruction scores, CT parameters of right ventricular dysfunction and adverse clinical outcome. Eur J Radiol 2012;81:3592-3597

11. Im DJ, Hur J, Han KH, Lee HJ, Kim YJ, Kwon W, et al. Acute pulmonary embolism: retrospective cohort study of the predictive value of perfusion defect volume measured with dual-energy CT. AJR Am J Roentgenol 2017;209:1015-1022

12. Weidman EK, Plodkowski AJ, Halpenny DF, Hayes SA, Perez-
Johnston R, Zheng J, et al. Dual-energy CT angiography for detection of pulmonary emboli: incremental benefit of iodine maps. Radiology 2018;289:546-553

13. Leithner D, Wichmann JL, Vogl TJ, Trommer J, Martin SS, Scholtz JE, et al. Virtual monoenergetic imaging and iodine perfusion maps improve diagnostic accuracy of dualenergy computed tomography pulmonary angiography with suboptimal contrast attenuation. Invest Radiol 2017;52:659665

14. Baek S, Park SH, Won E, Park YR, Kim HJ. Propensity score matching: a conceptual review for radiology researchers. Korean J Radiol 2015;16:286-296

15. Austin PC. Balance diagnostics for comparing the distribution of baseline covariates between treatment groups in propensity-score matched samples. Stat Med 2009;28:30833107

16. Kang DK, Thilo C, Schoepf UJ, Barraza JM Jr, Nance JW Jr, Bastarrika $\mathrm{G}$, et al. CT signs of right ventricular dysfunction: prognostic role in acute pulmonary embolism. JACC CardiovasC Imaging 2011;4:841-849

17. Furlan A, Aghayev A, Chang CC, Patil A, Jeon KN, Park B, et al. Short-term mortality in acute pulmonary embolism: clot burden and signs of right heart dysfunction at CT pulmonary angiography. Radiology 2012;265:283-293

18. Schoepf UJ, Kucher N, Kipfmueller F, Quiroz R, Costello $P$, Goldhaber SZ. Right ventricular enlargement on chest computed tomography: a predictor of early death in acute pulmonary embolism. Circulation 2004;110:3276-3280

19. Lu MT, Cai T, Ersoy H, Whitmore AG, Quiroz R, Goldhaber SZ, et al. Interval increase in right-left ventricular diameter ratios at CT as a predictor of 30-day mortality after acute pulmonary embolism: initial experience. Radiology 2008;246:281-287

20. Choi KJ, Cha SI, Shin KM, Lim J, Yoo SS, Lee J, et al. Prognostic implications of computed tomographic right ventricular dilation in patients with acute pulmonary embolism. Thromb Res 2014;133:182-186

21. Trujillo-Santos J, den Exter PL, Gómez V, Del Castillo H, Moreno $\mathrm{C}$, van der Hulle $\mathrm{T}$, et al. Computed tomographyassessed right ventricular dysfunction and risk stratification of patients with acute non-massive pulmonary embolism: systematic review and meta-analysis. J Thromb Haemost 2013;11:1823-1832

22. Becattini C, Agnelli G, Germini F, Vedovati MC. Computed tomography to assess risk of death in acute pulmonary embolism: a meta-analysis. Eur Respir J 2014;43:1678-1690

23. Goo HW, Goo JM. Dual-energy CT: new horizon in medical imaging. Korean J Radiol 2017;18:555-569

24. Kim EY, Seo JB, Oh SY, Lee CW, Hwang HJ, Lee SM, et al. Assessment of perfusion pattern and extent of perfusion defect on dual-energy CT angiography: correlations between the causes of pulmonary hypertension and vascular parameters. Korean J Radiol 2014;15:286-294

25. Miniati M, Monti S, Bottai M, Scoscia E, Bauleo C, Tonelli L, et al. Survival and restoration of pulmonary perfusion in a long- 
term follow-up of patients after acute pulmonary embolism. Medicine (Baltimore) 2006;85:253-262

26. Azarian R, Wartski M, Collignon MA, Parent F, Hervé P, Sors $H$, et al. Lung perfusion scans and hemodynamics in acute and chronic pulmonary embolism. J Nucl Med 1997;38:980-983
27. Hoey ET, Mirsadraee S, Pepke-Zaba J, Jenkins DP, Gopalan $D$, Screaton NJ. Dual-energy CT angiography for assessment of regional pulmonary perfusion in patients with chronic thromboembolic pulmonary hypertension: initial experience. AJR Am J Roentgenol 2011;196:524-532 